\title{
The role of spatial integration in the perception of surface orientation with active touch
}

\author{
Christos D. Giachritsis and Alan M. Wing \\ University of Birmingham, Birmingham, England \\ AND \\ Paul G. Lovell \\ University of Bristol, Bristol, England
}

\begin{abstract}
Vision research has shown that perception of line orientation, in the fovea area, improves with line length (Westheimer \& Ley, 1997). This suggests that the visual system may use spatial integration to improve perception of orientation. In the present experiments, we investigated the role of spatial integration in the perception of surface orientation using kinesthetic and proprioceptive information from shoulder and elbow. With their left index fingers, participants actively explored virtual slanted surfaces of different lengths and orientations, and were asked to reproduce an orientation or discriminate between two orientations. Results showed that reproduction errors and discrimination thresholds improve with surface length. This suggests that the proprioceptive shoulder-elbow system may integrate redundant spatial information resulting from extended arm movements to improve orientation judgments.
\end{abstract}

In perception, increasing spatial information about the property of an object or event often improves the accuracy and/or reliability of the percept. Findings from vision research suggest that restricting spatial information of static and dynamic stimuli can affect the perception of object properties and events. For instance, Westheimer and Ley (1997) have found that perception of line orientation, in the human fovea, improves with length: Discrimination of the orientation of a linear segment, relative to horizontal, improves with length and reaches an optimum when the line is about $0.5^{\circ}$ long. Moreover, steering ability and heading judgments have been found to be affected when optic flow information is limited. For instance, restricting visibility to the nearest $21 \mathrm{~m}$ results in the deterioration of a driver's steering ability, which is attributed to misperception of the radial structure of the optic flow (McLean \& Hoffman, 1973). In the laboratory, research has shown that manipulating the density of random-dot kinematograms affects heading judgments; denser optic flows tend to produce more accurate heading judgments (Warren \& Hannon, 1990; Warren, Mestre, Blackwell, \& Morris, 1991; Warren, Morris, \& Kalish, 1988). It was suggested that the visual system makes use of redundant optic flow information to improve heading judgments (Warren et al., 1991). These findings imply that the visual system may use spatial integration to increase the reliability of spatial information in order to decrease the uncertainty of the perception of object properties, such as edge orientation, and navigation events, such as steering and heading.
Haptics research has also shown that, under certain conditions, integration of spatial information can improve the perception of shape with active touch. For example, it has been found that shape recognition by the blind is better than that by the sighted because of the use of four rather than two fingers (Davidson, 1972; Davidson \& Whitson, 1974). It was suggested that multifinger contact was a better exploration method because it allowed a global sensing that could capture all the important aspects of the stimulus instantaneously. It seems that increasing overlapping sequential spatial integration may improve shape perception. In contrast, Loomis, Klatzky, and Lederman (1991) found that limited overlapping sequential spatial integration of tactile information did not affect shape recognition: Unimanual tactile recognition of objects represented by 2-D line drawings was not improved by doubling the receptive field from one finger (index) to two adjacent fingers (index and middle). These studies on active touch provide some contradictory results on spatial integration. In the following experiments, we addressed the problem of spatial integration in surface orientation in a manner closer to that employed by Westheimer and Ley (1997), since surface length was systematically varied. We expected that increasing spatial integration by exploring longer surfaces would increase the kinesthetic and proprioceptive signals received from the shoulder-elbow system and, therefore, improve judgments of surface orientation.

Research has shown that proprioceptive information from the shoulder and elbow system could be affected by

C. D. Giachritsis, c.giachritsis@bham.ac.uk 
a number of factors, including type of movements (i.e., passive or active) and gravitational forces. Absolute sensitivity to proprioceptive information from the shoulder and elbow seems to differ between tasks involving passive and active arm movements. For instance, during passive movements of a relaxed arm, the shoulder and elbow joints are very sensitive in detecting angular displacements; thresholds for the shoulder and elbow joints were found to be between $2^{\circ}$ at $0.2^{\circ} / \mathrm{sec}$ and $0.1^{\circ}$ at $3^{\circ} / \mathrm{sec}-80^{\circ} / \mathrm{sec}$, respectively (Hall \& McCloskey, 1983). Active contraction of the flexors during passive movement seemed to lower thresholds $\left(<0.3^{\circ}\right)$ for the detection of the direction of movement of the elbow joint, even at very slow speeds of $<0.05^{\circ} / \mathrm{sec}$ (Taylor \& McCloskey, 1992). However, other studies have shown that contraction of biceps and triceps during active movements increases the thresholds of detecting angular displacement at the elbow joint from $1^{\circ}$ to $2.5^{\circ}$ (Wise, Gregory, \& Proske, 1998). Therefore, it seems that proprioceptive signals from the shoulder and elbow joints may depend on the nature of the arm movements (active/passive) and on the condition of the muscles.

The larger angular displacements of the shoulder and elbow joints, which are required to explore longer surfaces, may increase the kinesthetic and proprioceptive signals but at the same time they could result in an increase of proprioceptive noise, due to gravitational torque acting on the joints. There are indications that an increase in the sense of effort, which results from an increase in the gravitational forces acting on the joints (e.g., Gooey, Bradfield, Talbot, Morgan, \& Proske, 2000), could increase the uncertainty of limb position. The effect of gravitational forces on proprioception has been shown in numerous experiments. For instance, Worringham and Stelmach (1985) found that manipulating gravitational torques affected participants' joint-angle matching performance. The error in producing a reference angle corresponded to the directional prediction of gravitational torques. In addition, Watson, Colebatch, and McCloskey (1984) found that when participants were asked to indicate the position of the left index finger with the right one, they took into account both position and force signals exerted by a spring on the right index finger. When contracting against a weak spring, participants could concentrate almost entirely on positional cues. As the spring became stiffer, the forces exerted on the wrist were harder to ignore, so the positioning of the limb was influenced by the achieved force. The role of gravitational forces in proprioception has also been shown in studies in which the human proprioceptive system had adapted to changes in gravitational forces (Roll et al., 1998; Soechting, 1982). Exploring longer surfaces requires extended arm movements involving greater muscular activity, which may increase the sense of effort, particularly near the horizontal and vertical, where gravitational effects are greater. For example, near the horizontal, the gravitational torque acting on the elbow and shoulder joints is maximum, but near the vertical, maximum effort is needed to lift the arm against gravity. The extra muscular activity required to explore long slants could increase the uncertainty of information about the orientations of the upper arm and lower arm (due to a low signal-to-noise ratio), and, therefore, could result in misjudgments of surface orientation.

Here, we investigate the effect of spatial integration on the perception of surface orientation, using proprioceptive information from the shoulder-elbow system. Participants judged the slant of surfaces with different lengths by moving their arms in and out in relation to the body. Our hypothesis was as follows: If increasing spatial integration improves the proprioceptive signal, this should result in more accurate orientation judgments, with longer rather than shorter surfaces. However, if movements that take the hand farther away from the body increase proprioceptive noise due to greater motor activity, the uncertainty of proprioceptive information should become greater, resulting in a deterioration of orientation judgments with surface length. A third possible outcome might be that increasing spatial exploration could increase proprioceptive noise at the same rate at which the proprioceptive signal increases, in which case we should expect the same absolute error and standard error of reproduced orientations across all surface lengths. In Experiment 1, we measured the accuracy of absolute estimates of surface orientation using a reproduction paradigm, and in Experiments 2 and 3 we measured the accuracy of relative estimates using a discrimination paradigm.

\section{EXPERIMENT 1 Reproduction of Surface Orientation}

Experiment 1 investigated the effect of spatial integration on absolute judgments of surface orientation using a reproduction method.

\section{Method}

Apparatus and Stimuli. A Phantom robot arm (1.5 Premium, SensAble Technologies) running under Windows XP was used to generate virtual surfaces at different orientations. The Phantom is a desktop position-sensing and force-feedback device with 3DOF for position and 3DOF for force; it exerts maximum and continuous forces of $8.5 \mathrm{~N}$ and $1.4 \mathrm{~N}$, respectively, and simulates shapes by coupling appropriate geometry and forces. Here, through a manufacturer-supplied thimble, the user was able to experience these forces in different positions in space and thereby effectively experience virtual slants.

The stimuli consisted of a narrow slanted surface of four different lengths $(35,70,140$, and $245 \mathrm{~mm})$ with damping and spring values set at 0 and 0.9 , respectively, giving the impression of a flat surface without introducing friction. The width of the surface was $2 \mathrm{~mm}$ and its sides were blocked with tilted walls in order to restrict sideways ( $x$-axis) movements of the hand. The two ends of the slant were marked by two curved edges to prevent a participant's hand from falling off the ends of the surface. The height of the edges was $30 \mathrm{~mm}$ with a corner radius of curvature of $10 \mathrm{~mm}$ (see Figure 1). The orientation of the surface varied randomly from $0^{\circ}$ to $90^{\circ}$, from trial to trial.

Procedure. Four men (including two of the authors) and two women, age range 20 to 56 years, were tested. Participants were seated directly in front of the Phantom and placed the left index finger in the thimble attached to the end of the Phantom arm. The choice of the exploring arm was arbitrary, since there is similar precision of information about the shoulder and elbow angles between the left and right arms (van Beers, Sittig, \& Denier van der Gon, 1998). Also, participants were instructed to keep their eyes closed throughout the 


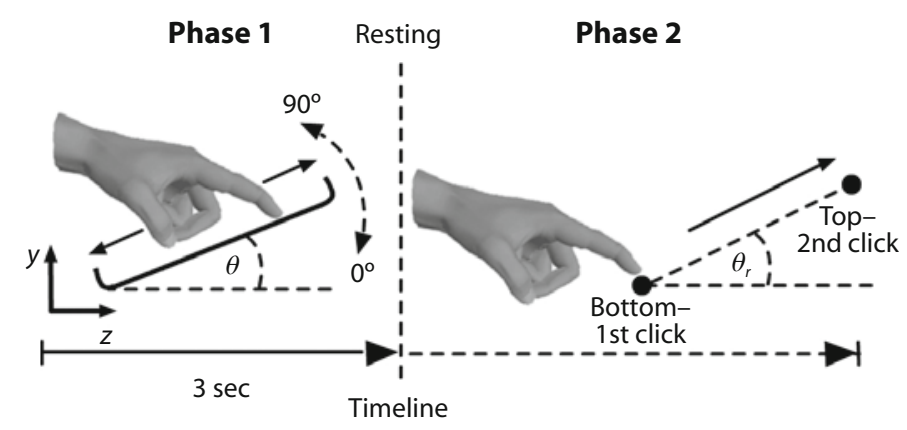

Figure 1. Testing procedure in Experiment 1. In the first phase, participants were asked to actively explore a narrow slanted surface by moving their index fingers repeatedly between the ends of the surface for 3 sec. In the second phase, the participants were asked to reproduce the surface orientation by indicating its two ends (starting from the bottom end) using a foot switch.

experiment. Each trial consisted of two phases. In the first phase, the apparatus guided the participants' left index fingers to the top of the slant. After the initial contact with the surface, the participants actively explored the surface by moving their fingers from one end of the slope to the other for $3 \mathrm{sec}$, which allowed them to sense the slant upward and downward two to four times, depending on the slant length. They were instructed to use arm movements and keep their fingers immobile during this phase. After $3 \mathrm{sec}$ of exploration, the apparatus moved their hands to the resting position, which was located in front of each participant just below chest level. In the second phase, the participants were asked to reproduce the slant by using the Phantom to indicate first its lower, then its upper, end. They pressed a foot switch to indicate each end when they were satisfied with their judgments (Figure 1). One participant reproduced 50 different orientations for each length; the rest reproduced 10 orientations per length. The experiment met the ethical requirements of the School of Psychology of the University of Birmingham and, including the practice session, lasted approximately 40 to $50 \mathrm{~min}$.

\section{Results}

The accuracy and reliability of slant reproduction were measured using linear regression (slope and intercept) and the coefficient of determination $r^{2}$, respectively. Ideal (or perfect) performance would result in regression slope of 1 , regression intercept of 0 , and coefficient of determination of 1. For example, Figure 2 shows how a regression line was used to measure the performance of participant N.K. under the 35- and 245-mm surface lengths $(n=10)$. The
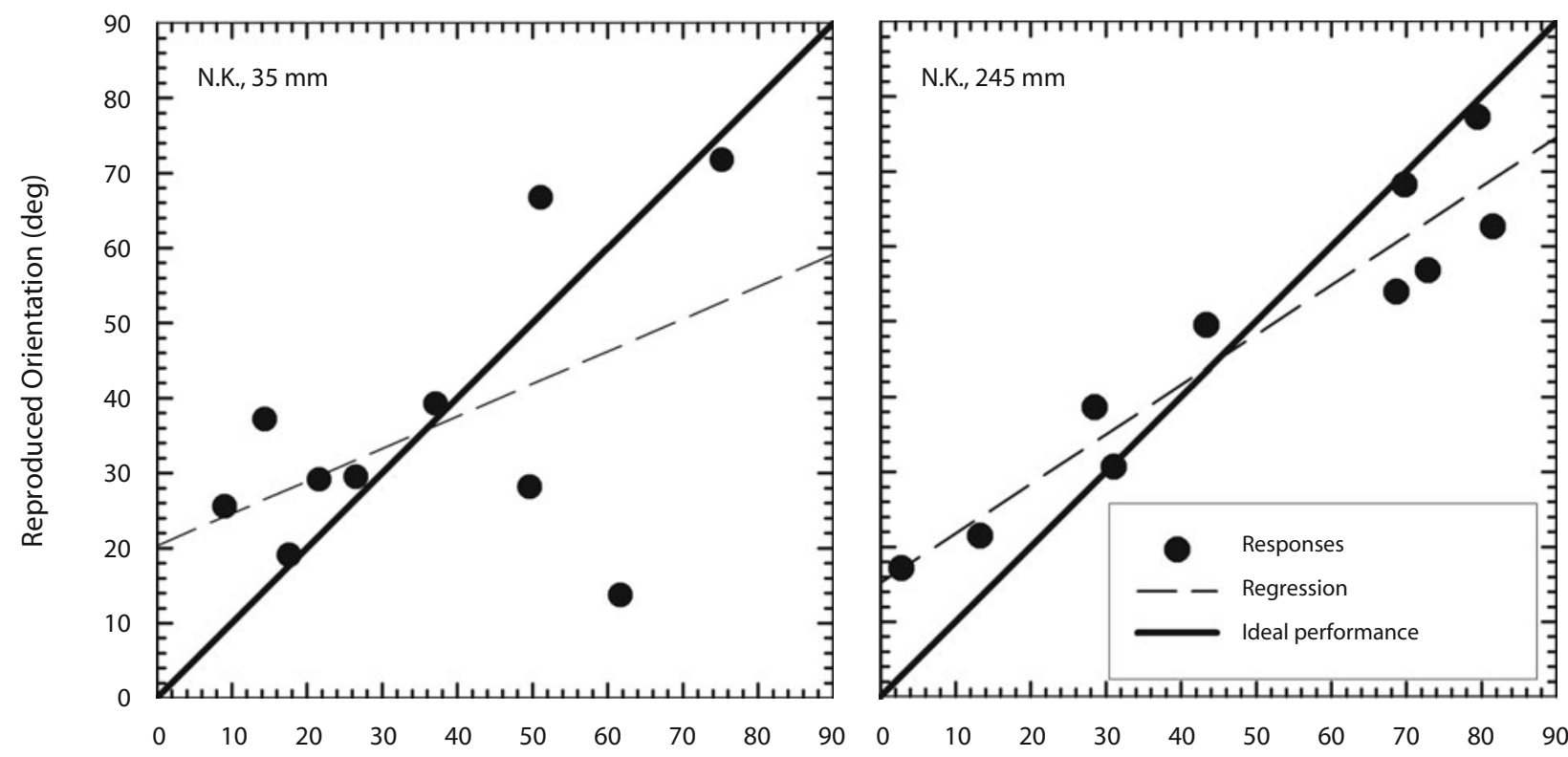

Explored Orientation (deg)

Figure 2. Accuracy and reliability of performance were measured with linear regression. Regression slope and intercept provided a measurement of the accuracy, and the correlation coefficient $r^{2}$ established the reliability of the estimates. This is an example of performance evaluation for participant N.K. $(n=10)$ under the two extreme lengths $(35$ and $245 \mathrm{~mm})$. The reproduced orientations were plotted against the actual orientations and a regression line was used to fit the data. 
A

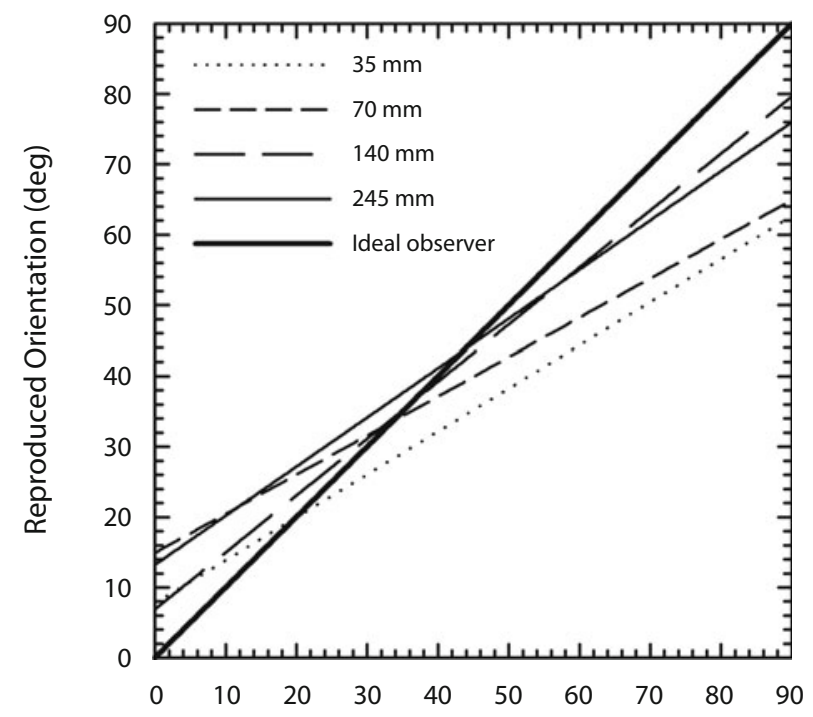

B

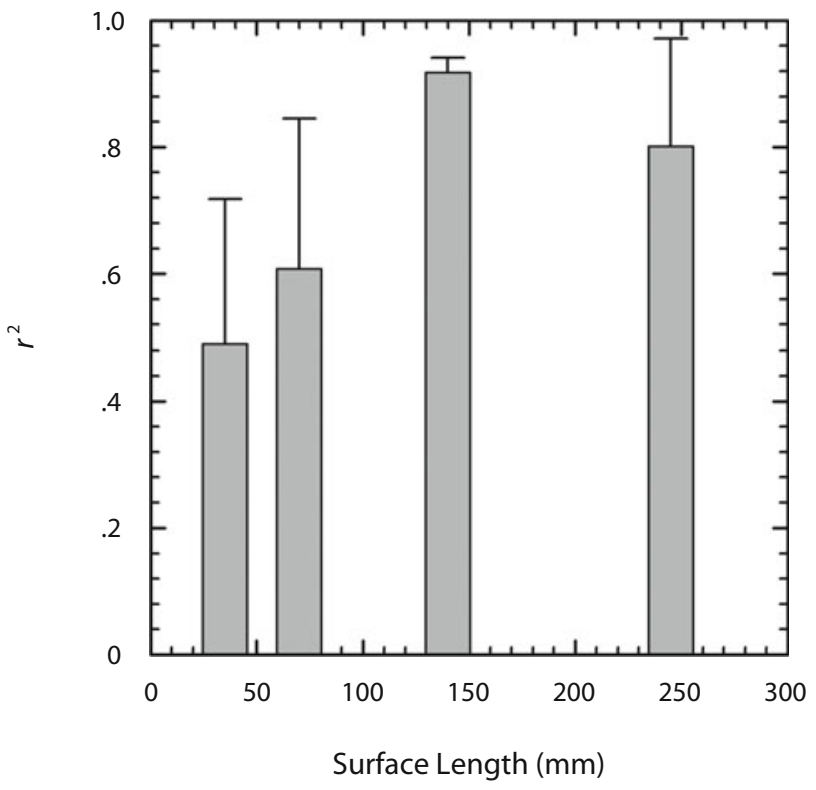

Figure 3. Overall accuracy of performance was summarized by regression lines (A), and overall reliability of performance was summarized by the coefficient of determination $r^{2}(B)$.

reproduced orientations were plotted against the actual surface orientations, then a linear regression was fitted to the data. Reproductions of the actual surface orientation were more accurate and more reliable with the $245-\mathrm{mm}$ length than with the 35-mm one.

Figure $3 \mathrm{~A}$ shows the accuracy of the overall performance expressed as a regression line for each surface length. Overall regression lines were generated by taking the average regression slope and intercept of the individual regression lines $(n=6)$. From this plot it can be seen that the slopes of 140- and 245-mm lengths are closer to unity than are those of 70- and 35-mm lengths; this indicates that performance improves with greater lengths. However, the intercepts do not show a similar trend. Also, the coefficient of determination $r^{2}$ tends to improve with surface length (Figure 3B). The low-regression slopes $(<1)$ and the high-regression intercepts $(>0)$, across all surface lengths, indicate that the participants tended to overestimate orientations near the horizontal and underestimate them near the vertical. A repeated measures ANOVA showed no statistically significant effect of the surface length on the regression slope or intercept. However, the increase of coefficient of determination $r^{2}$ with surface length was statistically significant $[F(3,15)=5.064, p=$ .013].

Figure 4 shows (A) individual and (B) overall performance for the absolute error (top panel) and the standard error of reproduced orientations (bottom panel) as functions of surface length. The absolute error of reproduced orientation is defined as the absolute difference between the reproduced and the actual orientation, and therefore measures the accuracy of performance. The standard error of reproduced orientations describes the standard deviation of the reproductions from the regression line, and therefore measures the accuracy of predictions (i.e., how accurately the regression line can predict reproduced orientations). The individual errors are produced by averaging errors from all trials, and the overall errors are produced by averaging individual errors. Both absolute and standard errors tend to reduce with surface length, indicating an improvement of perception of surface orientation with surface length. In general, greater lengths tend to produce smaller errors. A repeated measures ANOVA showed a statistically significant reduction in both absolute $[F(3,15)=22.458, p \approx .000]$ and standard $[F(3,20)=$ $17.494, p \approx .000]$ errors.

Reproduced length was also plotted against actual length (Figure 5). A regression line with slope 0.66 and intercept 23.28, which fitted the overall data well $\left(r^{2}=.9998\right)$, indicated that participants tended to reproduce lengths in a linear fashion. However, they did not reproduce lengths on a one-to-one basis. Participants also tended to underestimate greater lengths, in line with previous findings (Armstrong \& Marks, 1999).

It was observed that 2 out of 3 participants who reproduced the greatest lengths at $245 \mathrm{~mm}$ also reproduced the surface orientation more accurately. At 245-mm surface length, participant C.D.G. reproduced orientations resulting in an absolute error of $7.3^{\circ}$ and a mean length of $213.3 \mathrm{~mm}$, and participant C.E.G. reproduced orientations with an absolute error of $5.9^{\circ}$ and a mean length of $198.73 \mathrm{~mm}$. At this length, 4 out of 6 participants showed a very strong positive correlation $\left(r^{2}=.9974\right)$ between reproduced length and reproduced orientation. There was no correlation between reproduced length error and reproduced absolute orientation error at 35-mm surface length. 
A Individual Performance $(n=6)$
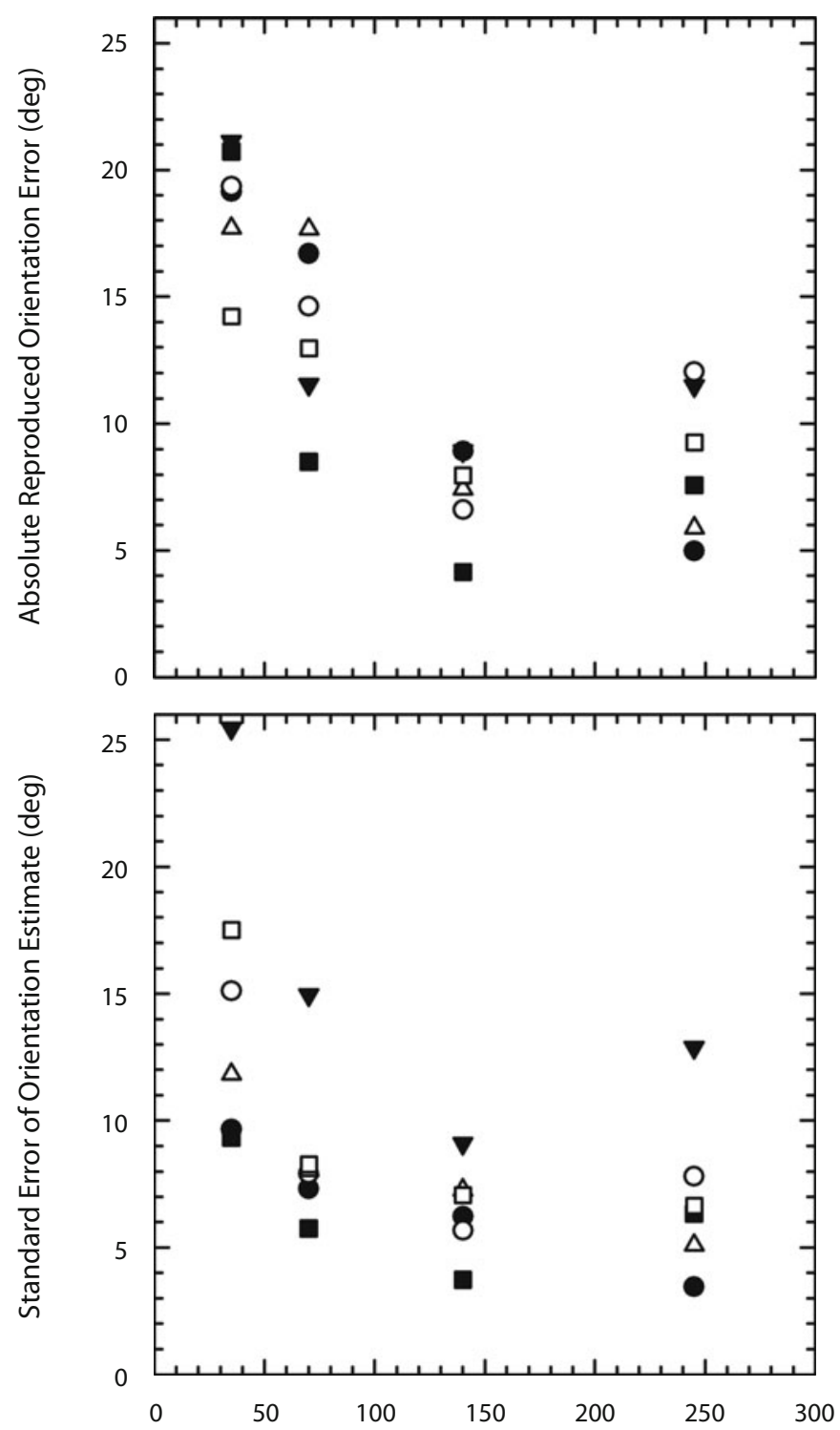

B

Overall Performance
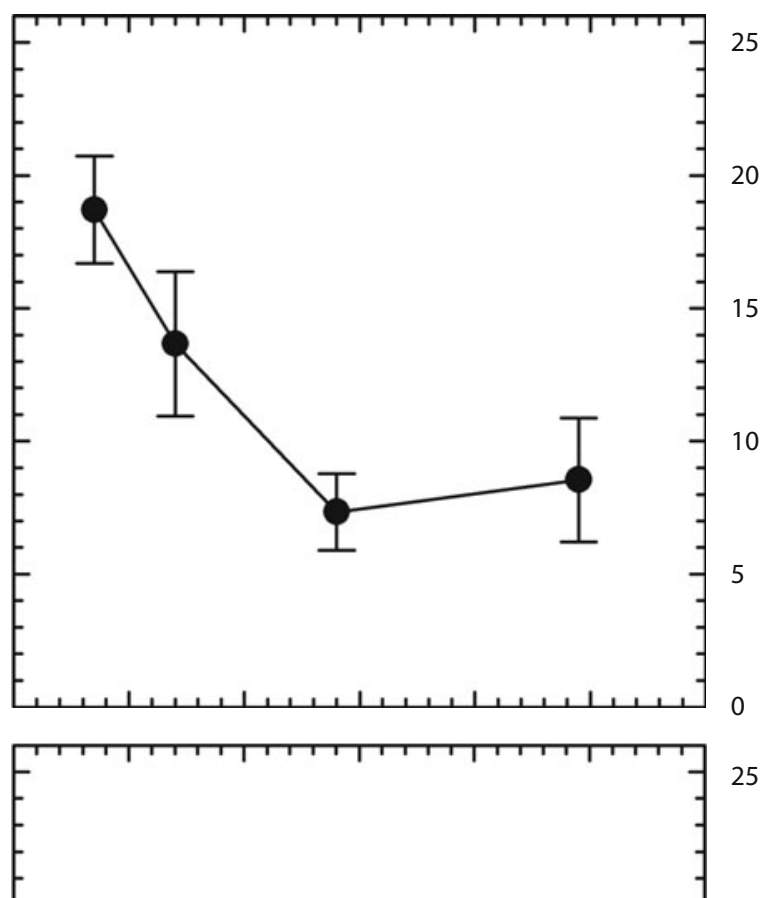

20

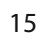

10

5

0

300

Surface Length $(\mathrm{mm})$

Figure 4. Individual (A) and overall (B) means of the absolute error (top panels) and the standard error (bottom panels) of reproduced orientations. Error bars represent $95 \%$ confidence limits.

This may suggest that exact replication of the surface could help in improving orientation estimates.

\section{Discussion}

Results from Experiment 1 showed that participants are better in reproducing the orientation of longer than of shorter surfaces. Both absolute error and standard error of reproduced orientations were reduced with surface length. This suggests that reliability of proprioceptive information may increase with increasing spatial integration. The proprioceptive system may be able to make use of redundant information resulting from extending arm movements in order to increase accuracy of slant estimates.

When participants reproduced lengths, they tended to overestimate shorter lengths and underestimate greater lengths. Misjudgments of linear extents have been found to be a common phenomenon in studies with active touch. For instance, Cheng (1968) and Armstrong and Marks (1999) found that participants overestimated radial lengths (compared with tangential lengths) located on the horizontal plane. Moreover, Henriques, Flanders, and Soechting (2004) observed that when participants were asked to reproduce quadrilateral shapes and open sequences of 
Individual Performance $(n=6)$

\section{है}

है
300
250

150

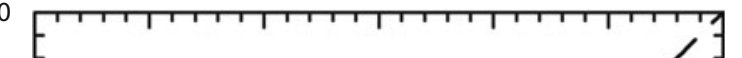

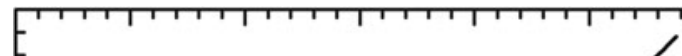

50

0

0

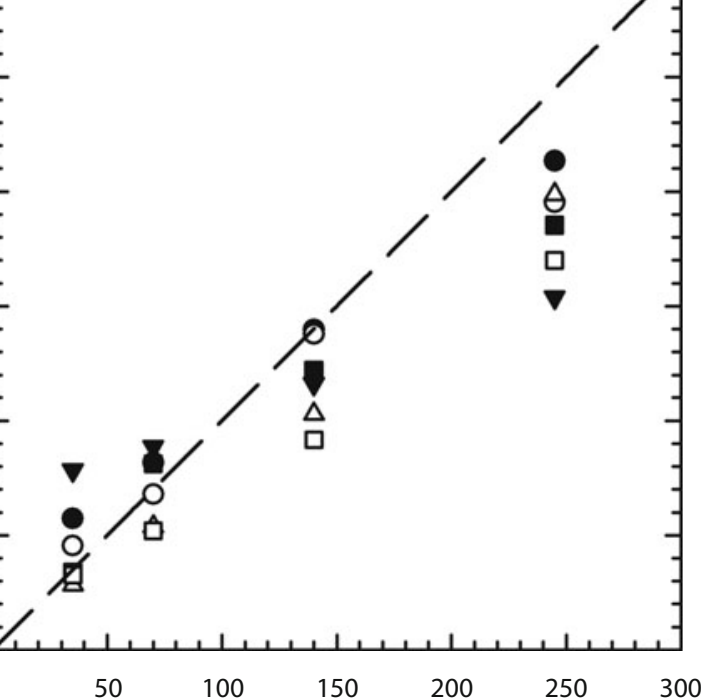

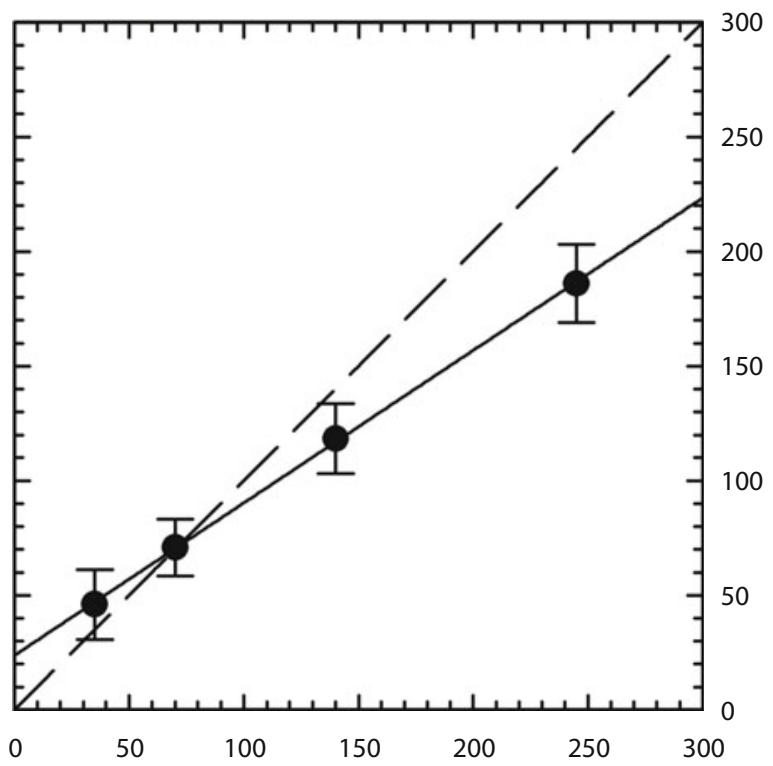

Actual Length (mm)

Figure 5. Individual and mean reproduced lengths plotted against actual lengths. Participants tended to reproduce lengths in a linear way, but not on a one-to-one basis. Error bars represent $95 \%$ confidence limits.

serially connected segments, they tended to overestimate shorter segments and underestimate longer segments. A plausible explanation for overestimating short lengths and underestimating long ones could be that the elbowshoulder system may be more frequently used to explore medium-sized objects, a familiarity which may have tuned it to perceive more accurately intermediate lengths rather than shorter or greater ones. Small objects could be more effectively explored using proprioceptive information from the finger joints, and large objects could be more frequently explored using both hands.

In conclusion, Experiment 1 showed that reproduction of surface orientation improved with surface length. This result could imply that reliability of proprioceptive information increases with increasing spatial integration. However, the greater variability of estimates with the 245-mm length, compared with the 140-mm length, may suggest that reproduction of orientations with longer surfaces could be affected by movement execution noise. In Experiments 2 and 3, we reduced the probable effects of execution noise by testing the ability of the participants to discriminate between different surface orientations instead of asking them to reproduce an orientation.

\section{EXPERIMENTS 2 AND 3 Discrimination of Surface Orientation}

In Experiment 1, we found that participants more accurately reproduced the orientation of longer than of shorter surfaces. However, reproducing surface orientation requires a voluntary arm movement that involves at least three stages: localization of target, movement planning, and movement execution. Movement execution could introduce noise with long linear paths, and this could have an effect on measuring slant perception with the longer surfaces. In Experiments 2 and 3, we wanted to investigate perception of surface orientation by excluding the possibility of movement execution noise affecting the judgments. Therefore, we tested sensitivity to discriminate surface orientation using a staircase method. In Experiment 2, participants were asked to judge the relative orientation of a surface with variable length $(35,70,140$, and $245 \mathrm{~mm})$ against a surface of standard length (i.e., $245 \mathrm{~mm}$ ). In Experiment 3 , participants were asked to judge the relative orientation of two surfaces with equal lengths. By testing participants with equal-length surfaces, we ensured that any possible effect of additional motor noise from exploring the standard $(245-\mathrm{mm})$ surface in every trial would be minimized.

\section{Method}

Apparatus and Stimuli. The apparatus and stimuli were the same as in Experiment 1.

Procedure. Five participants ( 4 men and 1 woman), age range 27 to 56 years, were used in these experiments. Two of the authors who participated in Experiment 1 were also tested in Experiments 2 and 3. Participants had their eyes closed throughout the experiment. A $2 \mathrm{AFC}$ adaptive method with a double staircase, for each surface length, was used to measure discrimination sensitivity to surface orientation. Each staircase approached the discrimination threshold from a different direction: The first tested sensitivity to "steeper" orientations, and the second to "shallower" ones. For both staircases the initial difference between the standard and test stimuli was $16^{\circ}$. Pilot studies had shown that, whereas participants were immediately able to tell the difference between the standard and a steeper test, they found it more difficult with shallower test 
slants. For this reason, we added $4^{\circ}$ at the lower limit of the "shallower" staircase. Therefore, the "steeper" and "shallower" values varied between $0^{\circ}$ and $16^{\circ}$ and between $-20^{\circ}$ and $0^{\circ}$ around the standard slant, respectively. The stimulus level ("shallower" or "steeper") reduced after three successive correct responses and increased after a single wrong response, thus determining the $79.4 \%$ threshold (Macmillan \& Creelman, 1991). The initial step size was $2^{\circ}$ and the minimum step size was $1^{\circ}$, reached after 2 reversals. The staircase stopped after 10 effective reversals had occurred, and the five means between the five pairs of the last 6 reversals were used to calculate the discrimination thresholds. All ethical requirements of the School of Psychology of the University of Birmingham were met.

Each trial was composed of three phases (Figure 6). In the first phase, the participants explored a surface of variable length $(35,70$, 140 , or $245 \mathrm{~mm}$ ), and in the second phase, they explored a standardlength surface of $245 \mathrm{~mm}$ in Experiment 2 and a surface of equal length in Experiment 3. In both phases, the exploration time was $3 \mathrm{sec}$. In the third phase, they specified whether the second (standard in Experiment 2) surface was "steeper" or "shallower" by using two foot-operated buttons. The orders of presenting the staircases and the standard and test stimuli were randomized and balanced, respectively. Both "steeper" and "shallower" thresholds were obtained in a single session that lasted approximately $1.5 \mathrm{~h}$.

\section{Results}

Discrimination thresholds were obtained for both "steeper" and "shallower" staircases, then the estimates were averaged to obtain a single threshold. In both experiments, individual and overall performance showed very similar trends: Sensitivity of relative orientation improved with surface length (Figures 7 and 8). Exploring longer surfaces apparently increased the reliability of proprioceptive information.

A repeated measures ANOVA performed on the averaged absolute thresholds showed that improvement of performance with surface length was statistically significant in both Experiment $2[F(3,12)=10.537, p=.001]$ and Experiment $3[F(3,12)=13.630, p \approx .000]$. Post hoc comparisons showed a statistically significant difference only between 35 and $245 \mathrm{~mm}$ in Experiment 2 and between $35 \mathrm{~mm}$ and all the others in Experiment 3.

\section{Discussion}

Experiments 2 and 3 have shown that discrimination thresholds for judging the relative orientation (steeper/ shallower) between two surfaces of unequal (Experiment 2) and equal (Experiment 3 ) lengths improve with surface length. This suggests that larger arm movements may provide more reliable proprioceptive and kinesthetic information than smaller movements, since there was a consistent decrease of thresholds across all surface lengths. In turn, this may suggest that the shoulder-elbow proprioceptive system could make use of redundant spatial information in order to improve perception of surface orientation.

\section{GENERAL DISCUSSION}

\section{Spatial Integration and Accuracy of Surface Orientation Judgments}

The present experiments have shown that increasing spatial integration improves judgments of surface orientation in the radial direction. In Experiment 1, absolute orientation estimates improved with surface length; absolute errors and standard errors of estimate reduced with surface length. However, slant reproduction with longer surfaces could be vulnerable to execution noise. There is some evidence that human participants tend to execute more curved movements when they are asked to move their arms to reach more distant targets (van Beers, Haggard, $\&$ Wolpert, 2004). However, the relevance of this finding here is questionable, since 2 of the 3 participants who reproduced the greatest lengths with the $245-\mathrm{mm}$ surface length also reproduced the most accurate orientations.

In Experiments 2 and 3, any possible reproduction noise was reduced by asking the participants to judge the relative orientation between two subsequently presented surfaces. We found that discrimination threshold improved with surface length. It seems that, as in some vision tasks (Warren et al., 1991; Westheimer \& Ley, 1997), the haptic system could make use of redundant spatial information,

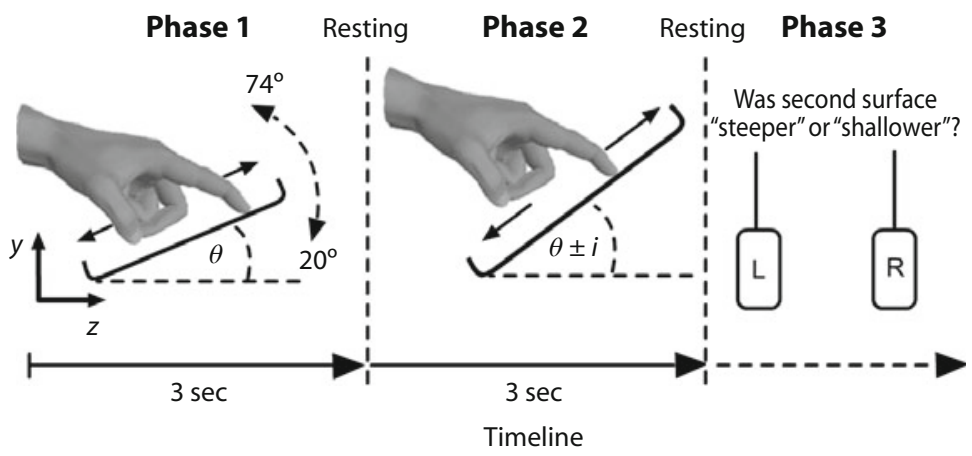

Figure 6. Testing procedure in Experiments 2 and 3. In Phase 1, participants explored a surface of 35-, 70-, 140-, or 245- $\mathrm{mm}$ length and $20^{\circ}-74^{\circ}$ orientation. In Phase 2, participants explored a standard surface of 245-mm length (Experiment 2) or an equal-length surface (Experiment 3), and orientation which was increased or decreased, depending on the staircase ("steeper" or "shallower"). In Phase 3, participants indicated (with the aid of two foot-operated buttons) whether the second surface was "steeper" or "shallower" than the first one. 
A

Individual Performance $(n=5)$
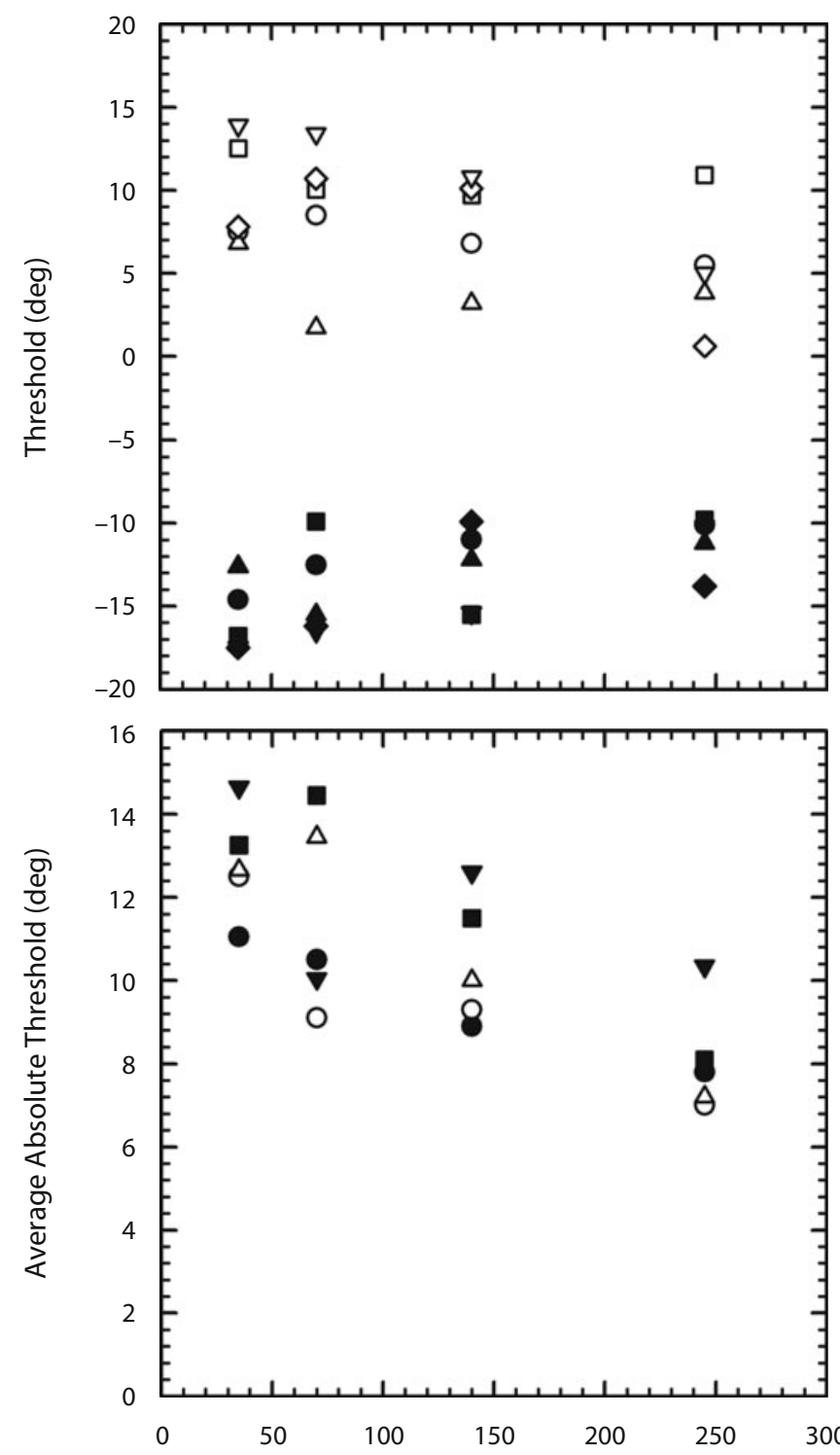

B

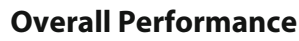

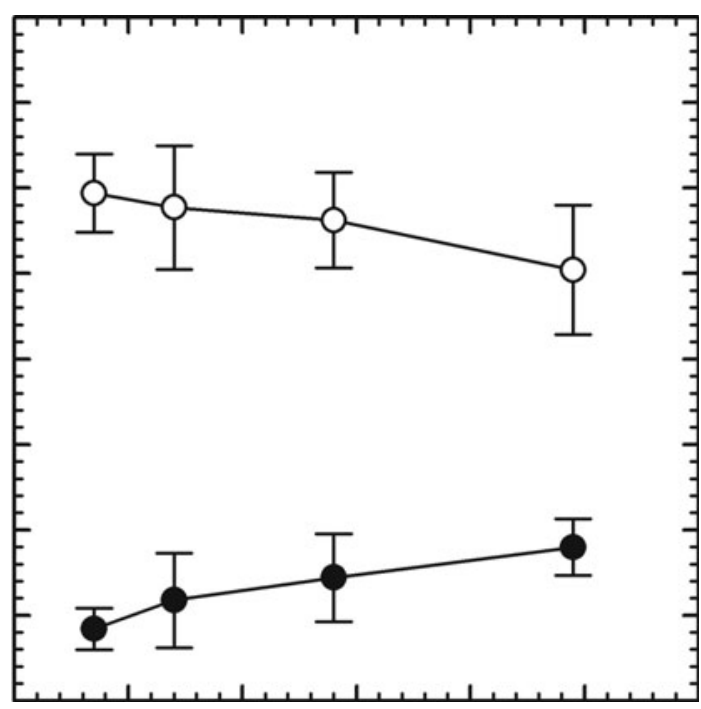

20

15

10

5

0

$-5$

$-10$

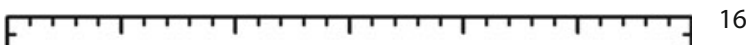

16

Surface Length (mm)

Figure 7. Shallower/steeper (top panels) and absolute discrimination (bottom panels) thresholds observed in Experiment 2. Individual (A) and overall (B) performances follow very similar trends: Discrimination between two surface orientations improves with the length of the test surface. Error bars represent $95 \%$ confidence limits.

through spatial integration, in order to reduce the uncertainty of the surface orientation percept. Increasing spatial integration may take advantage of redundant information (i.e., sensing more than two points that are necessary to estimate surface orientation) that could be used by the central nervous system to improve reliability of proprioceptive information from the shoulder-elbow system.

\section{Cumulative Integration of Local and Global Slant Information}

The present study has shown that slant perception is better with longer than with shorter surfaces. When we explore objects, we do so in a sequential fashion to obtain information about an object's size, shape, and orientation. During exploration, the haptic system may integrate both local and global proprioceptive and kinesthetic information to allow us to perceive both changes in local geometry and changes in the general direction of the fingertip. Therefore, slant perception could be based on the integration of local changes in movement direction into a global change by estimating the relative position between the starting point and all subsequent points.

Implementation of a cumulative integration model (see the Appendix), in which surface slant is estimated by pro- 
A Individual Performance $(\boldsymbol{n}=5)$

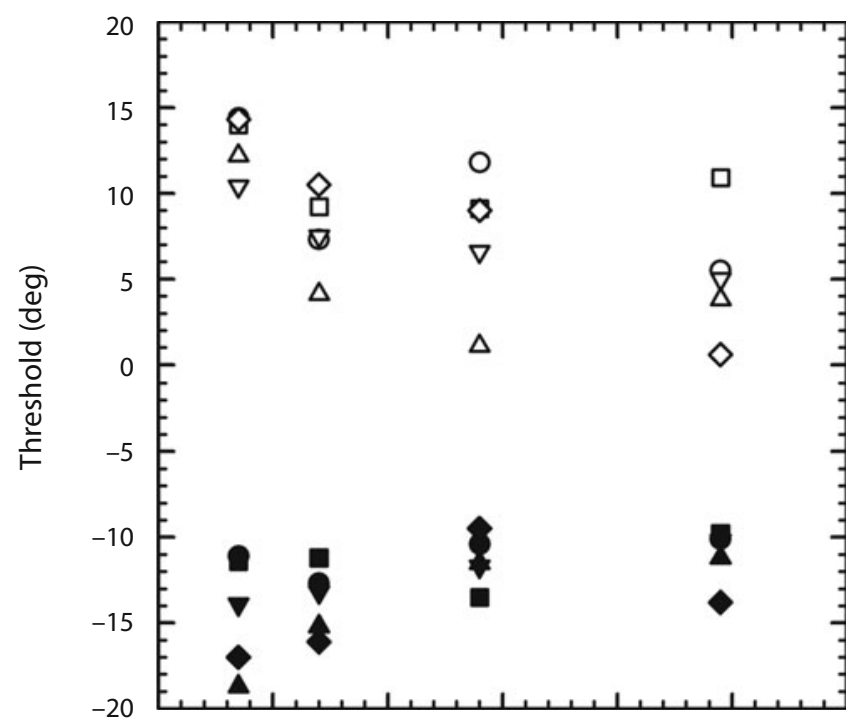

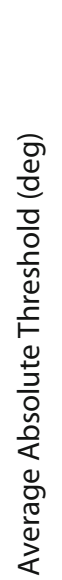

16

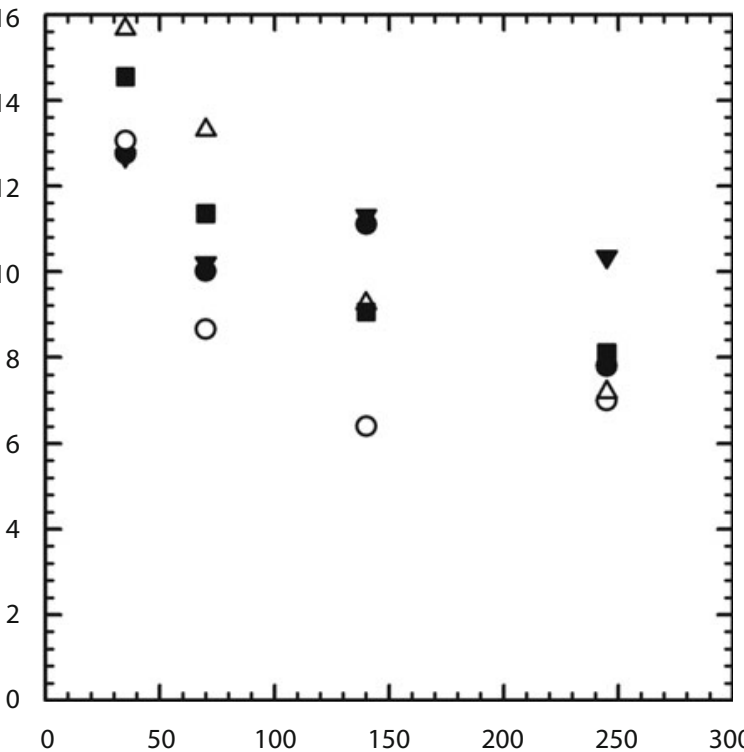

Surface Length (mm)

Overall Performance
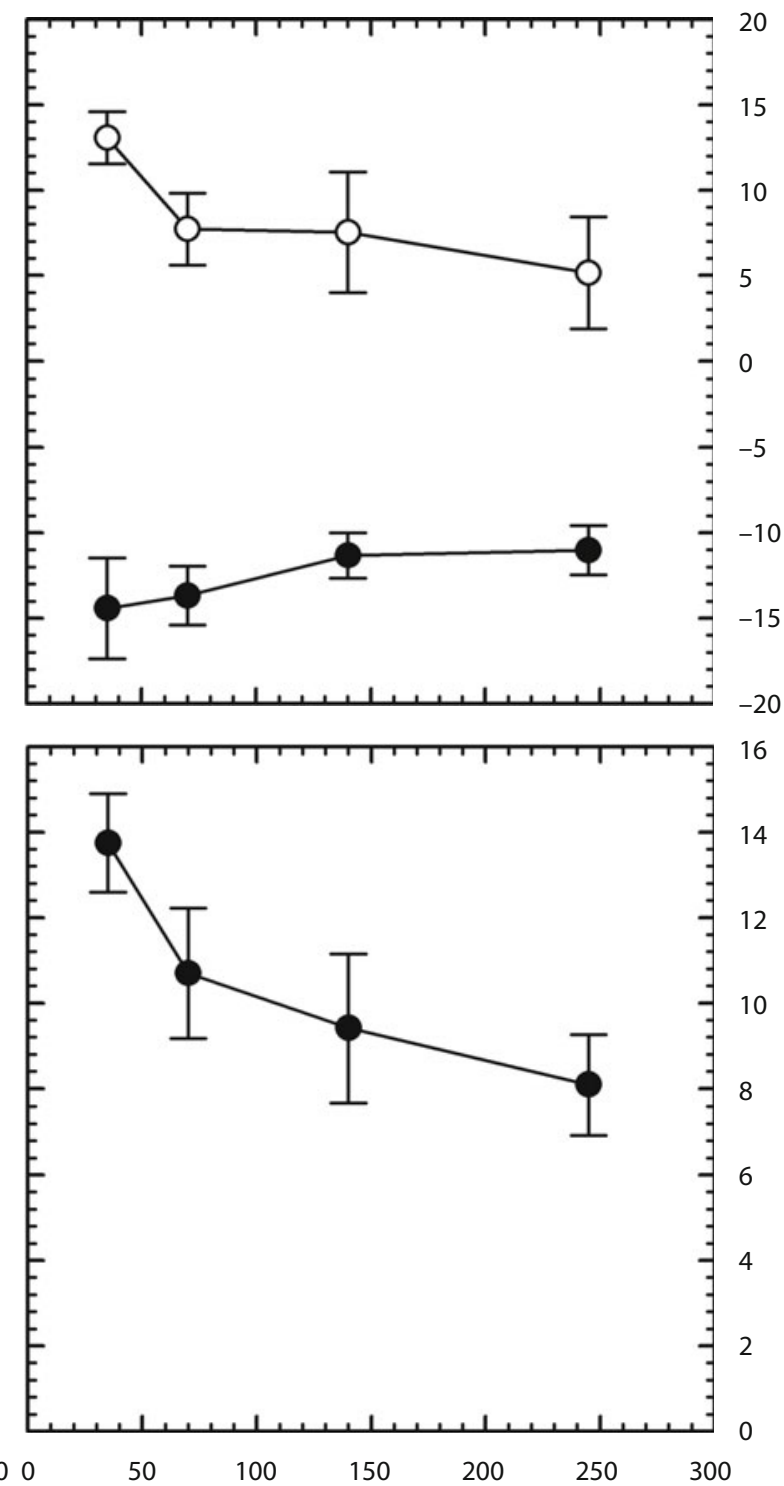

16

Figure 8. Shallower/steeper (top panels) and absolute discrimination (bottom panels) thresholds observed in Experiment 3. Individual (A) and overall (B) performances follow very similar trends: Discrimination between two surface orientations improves with surface length. Error bars represent $95 \%$ confidence limits.

gressively averaging all intermediate slant estimates (on a pair basis) between the starting point ( global information) and every subsequent point (local information), resulted in predictions qualitatively similar to observed data (Figure 9). However, the predicted and observed performances were quantitatively different. A possible explanation could be that the positional resolution of the haptic system is not constant but varies depending on the distance from the body (Haggard, Newman, Blundell, \& Andrew, 2000) or the extent of arm movements. For example, brief arm movements may induce little proprioceptive noise, result- ing in more accurate position estimates, whereas extended hand movements may result in increasing proprioceptive noise due to increasing muscular activity. As a result, position resolution in brief arm movements may be better than in longer ones; this could have affected particularly long slants requiring extended arm movements. Increasing spatial exploration could improve information about the direction of arm movement, but at the same time it could increase proprioceptive noise due to increasing muscular activity. In addition to proprioceptive noise, localization and planning noise (van Beers et al., 2004) could also have 
A

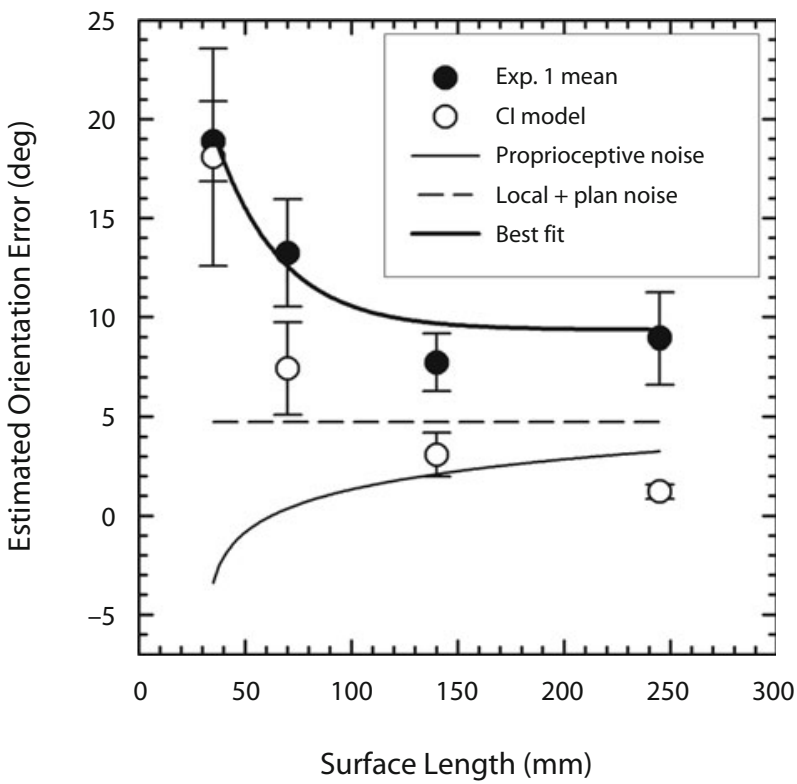

B

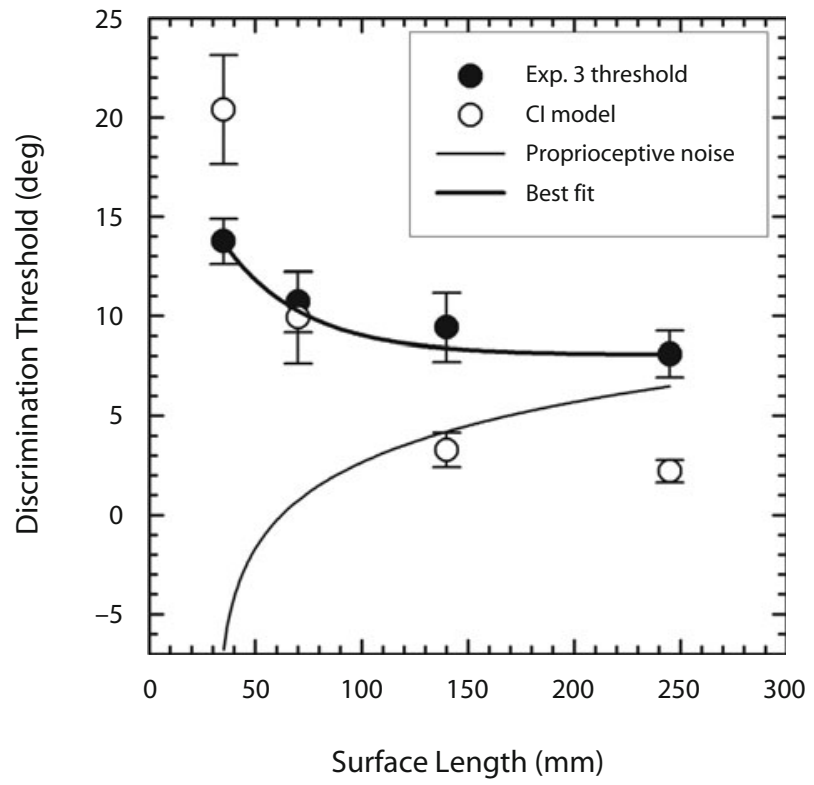

Figure 9. Comparing predictions of the cumulative integration (CI) model against human performance from Experiments 1 and 3. Human data are represented by symbols $\bullet$ and model predictions by $\circ$; error bars represent $95 \%$ confidence limits. (A) Predictions about absolute orientation errors (each data point is the average of the absolute values of 10 estimates) are compared against human orientation errors based on the reproduction paradigm from Experiment 1. (B) Discrimination thresholds from the CI model (each data bar is calculated on the basis of the 10 reversals from five staircases; see the Appendix for more detail) are compared against human discrimination thresholds from Experiment 3. Proprioceptive noise is represented by the thin continuous line, and localization and planning noise by the dotted line. Best fit is represented by the bold continuous line and is the result of adding proprioceptive noise and localization/planning noise to the model's predictions.

affected performance in the slant reproduction experiment. Introducing logarithmic proprioceptive noise and constant localization and planning noise seems to improve the model's performance remarkably (Figure 9).

\section{Perceptual and Cognitive Factors}

The results of the present experiments were explained on the basis of the effect of spatial integration on the accuracy and reliability of proprioceptive information and the possible execution noise on the judgments of relative and absolute surface orientation, but there are other factors that could have affected performance. Speed and duration of haptic exploration could be related to the accuracy of the perception of surface orientation. For instance, there is evidence that perception of haptic length is affected by the speed of exploration: Slower explorations result in greater apparent lengths, and faster explorations result in shorter apparent lengths (Armstrong \& Marks, 1999). In our experiments, participants were instructed to explore the surface freely, at their own pace. However, it was observed that participants explored the surfaces at very similar speeds. For example, in $3 \mathrm{sec}$, they completed about two exploration cycles (i.e., start-end-start), with the longest surface of $245 \mathrm{~mm}$ resulting in an exploration speed of approximately $327 \mathrm{~mm}^{-1}$. This could be considered to be an optimum speed, and applying faster or slower explorations could affect performance. Faster exploration speeds could corrupt proprioceptive information due to rapid changes in muscle length, which could lead to unreliable estimates of the orientations of the upper arm and the forearm by central mechanisms. On the other hand, slower speeds could increase the central sense of effort with long surfaces, which could result in even worse orientation judgments.

Similarly, duration and direction of exploration could have an effect on the perception of central effort and, subsequently, the judgment of surface orientation. In the present study, participants found that the duration of $3 \mathrm{sec}$ sufficed to form a percept of the surface orientation. However, longer explorations could increase the sense of effort, whereas shorter explorations (e.g., one complete cycle) could provide insufficient proprioceptive information. In addition, participants explored the surfaces in two directions, downward and upward. If effort and workload are affected by gravitational forces, we should expect that working with (downward exploration) or against (upward exploration) gravity could affect performance.

The direction of the movement over the surface could affect orientation judgments. There is evidence that radially orientated haptic lengths are overestimated compared with tangential lengths (Armstrong \& Marks, 1999; Cheng, 1968). Exploring radial lengths, particularly near the horizontal, increases the effect of gravity on the shoulder and elbow joints, and, consequently, the effect of sense of effort through increasing muscular activity. This increase in muscular activity could result in misjudgments of linear extents and orientations. 
In addition to these perceptual factors that could, potentially, play a role in the judgments of surface orientation, there are cognitive factors such as working memory and attention that could have affected the performance. In Experiments 2 and 3, participants were asked to judge the relative orientation of two surfaces presented sequentially. Even though the second surface was presented immediately after the exploration of the first surface, there was an approximately $1 \mathrm{sec}$ delay before the participants touched the second surface. Since judgment of the relative orientation of the second surface required access to the stored percept, the accuracy of the judgment should depend on the quality of this memory. To judge the relative orientation of the second surface, the participants could have followed at least two possible strategies: First, they could recall the first percept during the second exploration and make the judgment by comparing a real-time exploration and a recalled percept; second, they could complete the second exploration and make the judgment by comparing two recalled percepts. Both strategies are subject to flaws; following the first strategy, one would have to partially split one's attention between perceiving the real-time orientation and recalling as accurately as possible the percept stored in memory. Following the second strategy, one could devote all one's attention to the perception of the real-time orientation, but would have to compromise the quality of the first percept, since the time lapse between sensing the first orientation and making the judgment would have increased.

In conclusion, the present experiments have shown that exploring greater surface length can improve judgments of surface orientation; this suggests that increasing sequential spatial integration may improve the reliability of proprioceptive information from the shoulder-elbow system.

\section{AUTHOR NOTE}

This work was supported by the EU as part of the TOUCH-HAPSYS Project (IST-2001-38040), under FP5, and the IMMERSENCE Project (IST-4-027141), under FP6. Correspondence concerning this article should be addressed to C. D. Giachritsis, School of Psychology, University of Birmingham, Edgbaston, Birmingham B15 2TT, England (e-mail: c.giachritsis@bham.ac.uk).

\section{REFERENCES}

Armstrong, L., \& Marks, L. E. (1999). Haptic perception of linear extent. Perception \& Psychophysics, 61, 1211-1226.

Cheng, M. F. (1968). Tactile-kinesthetic perception of length. American Journal of Psychology, 81, 74-82.

Davidson, P. W. (1972). Haptic judgments of curvature by blind and sighted humans. Journal of Experimental Psychology, 93, 43-55.

Davidson, P. W., \& Whitson, T. T. (1974). Haptic equivalence matching of curvature by blind and sighted humans. Journal of Experimental Psychology, 102, 687-690.

Georgopoulos, A. P., Kalaska, J. F., Caminiti, R., \& Massey, J. T. (1982). On the relations between the direction of two-dimensional arm movements and cell discharge in primate motor cortex. Journal of Neuroscience, 2, 1527-1537.

Gooey, K., Bradfield, O., Talbot, J., Morgan, D. L., \& Proske, U. (2000). Effects of body orientation, load and vibration on sensing position and movement at the human elbow joint. Experimental Brain Research, 133, 340-348.

Haggard, P., Newman, C., Blundell, J., \& Andrew, H. (2000). The perceived position of the hand in space. Perception \& Psychophysics, 62, 363-377.

Hall, L. A., \& McCloskey, D. I. (1983). Detections of movements imposed on finger, elbow and shoulder joints. Journal of Physiology, 335, 519-533.

Henriques, D. Y. P., Flanders, M., \& Soechting, J. F. (2004). Haptic synthesis of shapes and sequences. Journal of Neurophysiology, 91, 1808-1821.

Kalaska, J. F., Caminiti, R., \& Georgopoulos, A. P. (1983). Cortical mechanisms related to the direction of two-dimensional arm movements: Relations in parietal area 5 and comparison with motor cortex. Experimental Brain Research, 51, 247-260.

Loomis, J. M., Klatzky, R. L., \& Lederman, S. J. (1991). Similarity of tactual and visual picture recognition with limited field of view. Perception, 20, 167-177.

Macmillan, N. A., \& Creelman, C. D. (1991). Detection theory: A user's guide. Cambridge: Cambridge University Press.

McLean, J. R., \& Hoffman, E. R. (1973). The effects of restricted preview on driver steering control and performance. Human Factors, $15,421-430$

Roll, R., Gilhodes, J. C., Roll, J. P., Popov, K., Charade, O., \& GuRfinKel, V. (1998). Proprioceptive information processing in weightlessness. Experimental Brain Research, 122, 393-402.

ScotT, S. H., \& KalasKa, J. F. (1997). Reaching movements with similar hand paths but different arm orientations. I. Activity of individual cells in motor cortex. Journal of Neurophysiology, 77, 826852.

Scott, S. H., Sergio, L. E., \& Kalaska, J. F. (1997). Reaching movements with similar hand paths but different arm orientations. II. Activity of individual cells in dorsal premotor cortex and parietal area 5 . Journal of Neurophysiology, 78, 2413-2426.

Soechting, J. F. (1982). Does position sense at the elbow reflect a sense of elbow joint angle or one of limb orientation? Brain Research, 248, 392-395.

TAYlor, J. L., \& McCloskey, D. I. (1992). Detection of slow movements imposed at the elbow during active flexion in man. Journal of Physiology, 457, 503-513.

van Beers, R. J., Haggard, P., \& Wolpert, D. M. (2004). The role of execution noise in movement variability. Journal of Neurophysiology, 91, 1050-1063.

van Beers, R. J., Sittig, A. C., \& Denier van der Gon, J. J. (1998) The precision of proprioceptive position sense. Experimental Brain Research, 122, 367-377.

van Beers, R. J., Sittig, A. C., \& Denier van der Gon, J. J. (1999). Localization of a seen finger is based exclusively on proprioception and on vision of the finger. Experimental Brain Research, 125, 43-49.

WARREN, W. H., JR., \& HANNON, D. J. (1990). Eye movements and optical flow. Journal of the Optical Society of America A, 7, 160-169.

Warren, W. H., Jr., Mestre, D. R., Blackwell, A. W., \& Morris, M. W. (1991). Perception of circular heading from optical flow. Journal of Experimental Psychology: Human Perception \& Performance, 17, 28-43.

Warren, W. H., Jr., Morris, M. W., \& Kalish, M. (1988). Perception of translational heading from optic flow. Journal of Experimental Psychology: Human Perception \& Performance, 14, 646-660.

Watson, J. D., Colebatch, J. G., \& McCloskey, D. I. (1984). Effects of externally imposed elastic loads on the ability to estimate position and force. Behavioural Brain Research, 13, 267-271.

Westheimer, G., \& Ley, E. J. (1997). Spatial and temporal integration of signals in foveal line orientation. Journal of Neurophysiology, 77, $2677-2684$.

Wiesendanger, M., Hummelsheim, H., \& Bianchetti, M. (1985). Sensory input to the motor fields of the agranular frontal cortex: A comparison of the precentral, supplementary motor and premotor cortex. Behavioural Brain Research, 18, 89-94.

Wise, A. K., Gregory, J. E., \& Proske, U. (1998). Detection of movements of the human forearm during and after co-contractions of muscles acting at the elbow joint. Journal of Physiology, 508, 325-330.

Worringham, C. J., \& Stelmach, G. E. (1985). The contribution of gravitational torques to limb position sense. Experimental Brain Research, 61, 38-42. 


\section{APPENDIX}

A Cumulative Integrator of Local and Global Slant Information

The accuracy of slant judgments from two adjacent surface points should depend on the accuracy with which the haptic system can resolve the position of the fingertip at these points. Let us assume that the position of the fingertip $P$ is resolved by central units that produce signals that can be described using a Gaussian-shaped function with the maximum likelihood being in their preferred position and that have overlapping receptive fields, which allows coactivation of neighboring units specifying an intermediate position. ${ }^{\mathrm{A} 1}$ The size and shape of their receptive fields should depend on the vector of the error of resolving the position of the fingertip $\vec{P}_{\varepsilon}$. Here, we assume that the magnitude of $\vec{P}_{\varepsilon}$ is direction and position independent, which results in a receptive field with spherical shape ${ }^{\mathrm{A} 2}$ and constant error throughout the haptic space, ${ }^{\mathrm{A} 3}$ respectively (Figure A1A).

On the basis of these assumptions, the haptic system could estimate surface slant, $\Theta^{\prime}$, by progressively averaging, on a pair basis, all intermediate slant estimates between the starting point and every subsequent point (cumulative integration model); that is,

$$
\Theta^{\prime}=\frac{\frac{\frac{\theta_{0}^{\prime}+\theta_{1}^{\prime}}{2}+\ldots+\theta_{k-1}^{\prime}}{2}+\theta_{k}^{\prime}}{2},
$$

where $\theta_{k}^{\prime}$ is an intermediate slant estimate between the first estimated position $P_{0}^{\prime}$ and a subsequent position $P_{(k+1)}^{\prime}$, and $k=n-1$. In principle, the total number of activated units should depend on the surface length and its orientation (Figure A1B). Given a regular, rectangular grid of units, $n$ takes maxima values near the horizontal and vertical and minima values near $45^{\circ}$.

For the purpose of simulation, we applied the following constraints on the above general model. First, we took the magnitude of $\vec{P}_{\varepsilon}$ to be $8 \mathrm{~mm}$. In a proprioception task, van Beers, Sittig, and Denier van der Gon (1999) found that the mean variance of finger localization in the $x y$ plane is $134 \pm 8 \mathrm{~mm}^{2}$. If we take the diagonal of the square with an area of $134 \pm 8 \mathrm{~mm}^{2}$ to be the diameter of the hypothetical receptive field, we end up with a radius of $8.185 \pm 0.241 \mathrm{~mm}$, which gives a $\vec{P}_{\varepsilon}$ magnitude of approximately $8 \mathrm{~mm}$. Second, in our task, exploration of the slant required movement of the arm, which provided participants with kinesthetic feedback. When we execute a voluntary movement, proprioceptive (e.g., arm position) and kinesthetic (e.g., direction of movement) information is available. Whereas the error of the proprioceptive feedback could be in any direction (e.g., van Beers et al., 1999), the error of kinesthetic feedback may be in the general direction of the movement (e.g., van Beers et al., 2004). Knowing the general direction of movement could increase the probability of perceiving the next $P$ to be in the general direction of movement. One way to achieve this is by introducing a common border constraint between two successive receptive fields which reduces the chances of two successive positional errors, $P_{\varepsilon}$ and $P_{\varepsilon+1}$, resulting in a local orientation which is opposite to the general direction of movement - that is, $x_{\varepsilon}>x_{\varepsilon+1}$ and $y_{\varepsilon}<y_{\varepsilon+1}$, or $x_{\varepsilon}<x_{\varepsilon+1}$ and $y_{\varepsilon}>y_{\varepsilon+1}$. We used this information about the general direction of arm movement as a constraint on the overlapping characteristic of the receptive fields; that is, during active exploration receptive fields do not overlap, but they have common boundaries (Figure A1B). Third, $\vec{P}_{\varepsilon}$ was taken to be maximum and randomly located at the edge (after the kinesthetic constraint has been applied) of the receptive field of each activated unit (Figure A1B).

On the basis of these assumptions and constraints, we carried out two simulations to find the absolute errors and the $79.4 \%$ discrimination thresholds for each surface length. In the first simulation, 10 slant estimates per length were obtained for slants which varied randomly from $0^{\circ}$ to $90^{\circ}$. Then the absolute error was calculated by taking the absolute difference between the randomly generated slant and the model's estimated slant. In the second simulation, five discrimination thresholds per length were obtained using a staircase method with "standard" and "test" slants of equal length (as in Experiment 3). The "test" slant was always steeper than the "standard" slant, whose values varied randomly between $0^{\circ}$ and $70^{\circ}$. The initial relative value of the "test" slant was $+20^{\circ}$ and reduced or increased by a value which was based on the step size. The initial step size was $4^{\circ}$ and reduced by half after each reversal took place until it reached $1^{\circ}$. However, if - after a series of successful responses - the calculated angle difference between the "test" and "standard" for the next trial was $0^{\circ}$, the step size was reduced by half without waiting for a reversal to occur. This was done in order to avoid presentation of two identical slants. The angle difference was reduced every time the model gave three successive correct responses, but it was increased with a single wrong response. A correct response was taken to be the positive difference between the model's estimates of the "test" and "standard" slants. Each session terminated after 10 reversals, which were used to calculate the threshold in the same way as in Experiment 3. 


\section{APPENDIX (Continued)}
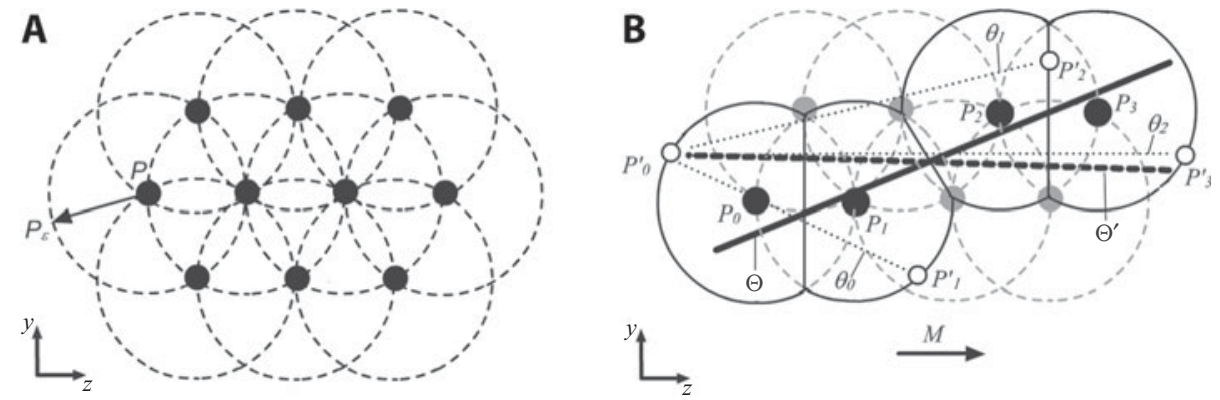

Figure A1. Schematic representation of how the haptic system could resolve spatial position (A) and estimate slant (B). The position of the fingertip in space could be resolved by position selective units, $P$, which have overlapping receptive fields with radii that equal the vector of the error of resolving $P, P_{\varepsilon}(\mathrm{A})$. Given such a system, an orientation estimate, $\Theta^{\prime}$, of the surface orientation, $\Theta$, could be obtained on the basis of all the estimated suborientations, $\theta_{k}^{\prime}$, between the estimated starting point $P_{0}^{\prime}$ and all subsequent estimated positions $P_{(k+1)}^{\prime}($ where $k=n-1)(\mathrm{B})$.

\section{NOTES}

A1. There is evidence that there are similar types of specialized units responding to passive (Wiesendanger, Hummelsheim, \& Bianchetti, 1985) and active arm movements (Georgopoulos, Kalaska, Caminiti, \& Massey, 1982; Kalaska, Caminiti, \& Georgopoulos, 1983) as well as to specific arm orientations (Scott \& Kalaska, 1997; Scott, Sergio, \& Kalaska, 1997), all of which could be involved in resolving the position of the fingertip.

A2. It should be noted that experiments on judgments of the lengths of haptic extents in the radial and tangential direction have shown that participants overestimate radially positioned extents and underestimate tangentially positioned extents. This suggests that the shape of the receptive field may be elongated spheroid rather than spherical (Armstrong \& Marks, 1999; Cheng, 1968).

A3. Again, it should be noted that there is evidence that proprioception accuracy of the shoulder-elbow system may vary with distance of the hand from the body (Haggard et al., 2000). This may indicate that $\vec{P}_{\varepsilon}$ could increase with distance from body.

(Manuscript received March 16, 2009;

revision accepted for publication April 15, 2009.) 